\title{
Prognostic Factors in the Decision-Making Process for Sigmoid Volvulus. Results of a Single-Centre Retrospective Cohort Study.
}

\section{Zoe Slack}

Oxford University Hospitals NHS Trust: Oxford University Hospitals NHS Foundation Trust

\section{Mohamed Shams}

Oxford University Hospitals NHS Trust: Oxford University Hospitals NHS Foundation Trust

\section{Raheel Ahmad}

Oxford University Hospitals NHS Trust: Oxford University Hospitals NHS Foundation Trust

\section{Roshneen Ali}

Oxford University Hospitals NHS Trust: Oxford University Hospitals NHS Foundation Trust

\section{Diandra Antunes}

Oxford University Hospitals NHS Trust: Oxford University Hospitals NHS Foundation Trust

Abhishek Dey

Oxford University Hospitals NHS Trust: Oxford University Hospitals NHS Foundation Trust

\section{Mahul Patel}

Oxford University Hospitals NHS Trust: Oxford University Hospitals NHS Foundation Trust

\section{Amanda Shabana}

Oxford University Hospitals NHS Trust: Oxford University Hospitals NHS Foundation Trust

\section{Giles Bond-Smith}

Oxford University Hospitals NHS Trust: Oxford University Hospitals NHS Foundation Trust Giovanni Domenico Tebala ( $\square$ giovanni.tebala@ouh.nhs.uk)

Oxford University Hospitals NHS Trust: Oxford University Hospitals NHS Foundation Trust https://orcid.org/0000-0001-7152-4096

\section{Research Article}

Keywords: sigmoid volvulus, frailty, emergency colon resection

Posted Date: March 16th, 2021

DOI: https://doi.org/10.21203/rs.3.rs-302366/v1

License: (c) (i) This work is licensed under a Creative Commons Attribution 4.0 International License. Read Full License 
Version of Record: A version of this preprint was published at BMC Surgery on March 14th, 2022. See the published version at https://doi.org/10.1186/s12893-022-01549-4. 


\section{Abstract}

BACKGROUND: Sigmoid volvulus is a common cause of emergency surgical admission. It often affects older males who are institutionalized and are less suitable surgical candidates. Definitive treatment is surgical, but first line treatment is via endoscopic devolution with or without placement of a rectal tube. After non-operative management recurrence is likely and carries a high mortality, therefore an early surgical approach may be considered in patients who are fit for surgery. We have retrospectively analyzed a cohort of patients with sigmoid volvulus in order to clarify if and when a more aggressive management is indicated.

METHODS: We have reviewed data on admitted patients diagnosed with sigmoid volvulus over a 2-year period. Demographic, clinical data, morbidity and mortality were recorded in a database. The primary endpoint was patient survival. Secondary endpoint was the estimation of the factors that condition surgical choice.

RESULTS: We analysed 332 admission of 78 patients. $39.7 \%$ of patients underwent surgical resection. The average survival was $54.9 \pm 8.8$ months from the first hospitalization, irrespective of the treatment. Long-term survival was positively influenced by being female, having a low "social score", a younger age and surgery. Multivariate analysis showed that only being female and surgery were independently associated with better survival.

CONCLUSION: Early surgery may be the best approach in patients with recurrent sigmoid volvulus, as it ensures longer survival with a better quality of life, regardless of the patient's social and functional condition.

\section{Background}

Sigmoid volvulus is a result of a redundant segment of bowel twisting on its mesentery. The word "volvulus" originates from the Latin "volvere" meaning to twist. Von Rokitanski described it in his book "A Manual of Pathological Anatomy" in 1848, but it could have been described many centuries before. The Papyrus Ebers described "twist in a bowel" that if left untreated will rot and the abdomen will become stiff, distended and painful, unless treatment is given to evacuate the bowel immediately. [1]

In the western world sigmoid volvulus affects older males who are institutionalized and may have neurological deficit making them less preferable surgical candidates [2, 3]. Other pre-disposing factors include chronic constipation, neurological disease, anatomical predisposition and adhesions $[4,5]$. Complications include ischaemia, gangrene and perforation which can result in a surgical emergency. Definitive treatment is surgical, but first line treatment is via endoscopic devolution with or without placement of a rectal tube. After non-operative management recurrence occurs in $50-90 \%$ of cases $[3,4]$ carrying a mortality of $7 \%-20 \%$ [5-7]. Due to the high risk of recurrence, the American Society of Colon and Rectal Surgeons (ASCRS) publicized guidelines in 2016 stating that patients should undergo a colonic resection after an episode of volvulus [8]. However, the timing of this intervention remains 
controversial. Moreover, those patients are usually frail and with multiple co-morbidities, making them less preferable surgical candidates. For this reason, nowadays most patients with sigmoid volvulus are not offered a surgical resection and, despite multiple admissions, are always treated conservatively, with increased risk and discomfort for the patients and high cost for the society.

Aim of this pilot study was to identify the prognostic factors to be considered in the decision-making process in patients with sigmoid volvulus and to see if a more aggressive approach early in the clinical course, as suggested by clinical evidence and guidelines, may represent a benefit for those difficult patients. This paper would represent the basis for a future prospective study.

\section{Methods}

This retrospective cohort study was performed at the Surgical Emergency Unit of the Oxford University Hospitals NHS Foundation Trust. Electronic records of patients admitted for "volvulus" in a 2-year period were reviewed. The initial screening returned 130 patients, but data of 52 patients with discharge diagnosis of caecal volvulus (24), small bowel volvulus (16), gastric volvulus (3), transverse colon volvulus (1), diverticular stricture (1) and pseudo-obstruction/ileus (7) where excluded from the analysis. The final analysis was therefore conducted on 78 patients treated for sigmoid volvulus during 332 admissions. They were 55 men and 23 women, aged $73.6 \pm 14.5$ (range 26-97) at index admission.

For each patient the following parameters were collected at index admission: demographic data (age, gender, social circumstances), clinical data (past medical history, comorbidities, frailty, ASA score, performance status), volvulus treatment data (eventual surgery, number of previous admissions, surgical mortality, months between first admission and surgery), radiological findings (coffee bean sign at abdominal film, CT confirmation of sigmoid volvulus, 360-degree twist, signs of ischaemia at CT), laboratory findings (white cells count, neutrophils count, c-reactive protein).

Social circumstances were coded with a "Social Score": fully independent, supported by family, package of care, full-time care. The Clinical Frailty Scale was used to grade each patient's frailty [9]. Performance status was graded according to the World Health Organization scale: fully active, restricted in physically strenuous activity but ambulatory and able to carry out work of a light or sedentary nature, ambulatory and capable of all selfcare but unable to carry out any work activities, capable of only limited selfcare, completely disabled and totally confined to bed or chair, dead.

The outcome of each patient was initially assessed on the basis of his or her electronic records including records of consultations or investigations under other specialities. In the few cases where no recent entry was available on the electronic system, a round of phone calls was done to assess the status of the patient. For patients without a recent medical appointment and who could not be contacted by the telephone, the last entry was considered as censor date and status.

Primary endpoint of the study was actuarial survival. Secondary endpoints were the estimate of the risk of needing surgery in case of recurrent sigmoid volvulus and the identification of the factors favouring 
the surgical approach with the aim of providing a prognostic and decisional framework to guide the choice of the treatment on individual bases.

Data have been collected into an electronic database (MS Excel for Mac v.16.44) and analysed with two statistical packages (StatPlusMac v.7.3.32 and GNU-PSPP v.1.2.0). Distribution of each variable was initially tested for skewness and kurtosis. Comparisons of continuous variables were performed with the Student's T-test (normally distributed variables) or with the Mann-Whitney U-test (non-parametric distribution). Comparisons of categorical variables were performed with the Pearson's Chi-square test. Multivariate logistic regression analysis was conducted on the whole range of variables and then on those variables that had resulted most significant at univariate analysis with a stepwise approach. Survival analysis was performed with the Kaplan-Meier method and survival comparison was conducted with the log-rank test. Survival regression was conducted with the Cox Proportional-Hazards regression method. $\mathrm{P}<0.05$ was considered to be significant. Missing data have been excluded from the analysis.

This report has been prepared on the basis of the STROBE guidelines [10].

\section{Results}

Fifty-eight patients (74.4\%) had more than one admission for sigmoid volvulus. Average number of admissions per patient was $4.3 \pm 3.9$ (range $1-18$, median 3 ). Thirty-one patients underwent surgery $(39.7 \%)$. Univariate analysis of the factors predisposing to surgery is reported in Table 1. 
Table 1

Univariate analysis. * percentage within column; ** percentage within row

\begin{tabular}{|c|c|c|c|c|c|}
\hline Factor & & Overall * & Surgery ** & No surgery ** & $p$ \\
\hline Total & & 78 & $31(39.7 \%)$ & $57(60.3 \%)$ & \\
\hline \multirow[t]{2}{*}{ Gender } & M & $55(70.5 \%)$ & 18 (32.7\%) & $37(67.3 \%)$ & \multirow[t]{2}{*}{0.050} \\
\hline & $F$ & $23(29.5 \%)$ & $13(56.5 \%)$ & $10(43.5 \%)$ & \\
\hline \multirow[t]{4}{*}{ Social Score } & 0 & $32(42.7 \%)$ & $18(56.3 \%)$ & $14(43.7 \%)$ & \multirow[t]{4}{*}{0.149} \\
\hline & 1 & $8(10.7 \%)$ & $3(37.5 \%)$ & $5(62.5 \%)$ & \\
\hline & 2 & 14 (18.7\%) & $4(28.6 \%)$ & $10(71.4 \%)$ & \\
\hline & 3 & $21(28.0 \%)$ & $6(28.6 \%)$ & $15(71.4 \%)$ & \\
\hline \multirow[t]{4}{*}{ ASA score } & 1 & $1(1.3 \%)$ & $1(100 \%)$ & 0 & \multirow[t]{4}{*}{0.243} \\
\hline & 2 & $35(45.5 \%)$ & $17(48.6 \%)$ & $18(51.4 \%)$ & \\
\hline & 3 & $34(44.2 \%)$ & $10(29.4 \%)$ & $24(70.6 \%)$ & \\
\hline & 4 & $7(9.1 \%)$ & $3(42.9 \%)$ & $4(57.1 \%)$ & \\
\hline \multirow[t]{2}{*}{ Neurological disease } & Yes & $46(59.7 \%)$ & $17(37.0 \%)$ & $29(63.0 \%)$ & \multirow[t]{2}{*}{0.472} \\
\hline & No & $31(40.3 \%)$ & $14(45.2 \%)$ & $17(54.8 \%)$ & \\
\hline \multirow[t]{9}{*}{ Frailty score } & 1 & $7(9.3 \%)$ & $3(42.9 \%)$ & $4(57.1 \%)$ & \multirow[t]{9}{*}{0.748} \\
\hline & 2 & $10(13.3 \%)$ & $5(50.0 \%)$ & $5(50.0 \%)$ & \\
\hline & 3 & $13(17.33 \%)$ & $7(53.9 \%)$ & $6(46.1 \%)$ & \\
\hline & 4 & $8(10.7 \%)$ & $4(50.0 \%)$ & $4(50.0 \%)$ & \\
\hline & 5 & $3(4.0 \%)$ & $1(33.3 \%)$ & $2(66.7 \%)$ & \\
\hline & 6 & $13(17.3 \%)$ & $6(46.2 \%)$ & $7(53.9 \%)$ & \\
\hline & 7 & $18(24.0 \%)$ & $5(27.8 \%)$ & $13(72.2 \%)$ & \\
\hline & 8 & $2(2.7 \%)$ & 0 & $2(100 \%)$ & \\
\hline & 9 & $1(1.3 \%)$ & 0 & $1(100 \%)$ & \\
\hline \multirow[t]{4}{*}{ Performance status } & 0 & $9(11.8 \%)$ & $4(44.4 \%)$ & $5(55.6 \%)$ & \multirow[t]{4}{*}{0.299} \\
\hline & 1 & $14(18.4 \%)$ & $6(42.9 \%)$ & $8(57.1 \%)$ & \\
\hline & 2 & $13(17.1 \%)$ & $8(61.5 \%)$ & $5(38.5 \%)$ & \\
\hline & 3 & 27 (35.5\%) & 7 (25.9\%) & $20(74.1 \%)$ & \\
\hline
\end{tabular}




\begin{tabular}{|c|c|c|c|c|c|}
\hline Factor & & Overall * & Surgery ** & No surgery ** & $p$ \\
\hline & 4 & $13(17.1 \%)$ & $5(38.5 \%)$ & $8(61.5 \%)$ & \\
\hline \multirow[t]{2}{*}{ Coffee bean sign at AXR } & Yes & $3(4.2 \%)$ & $2(66.7 \%)$ & $1(33.3 \%)$ & \multirow[t]{2}{*}{0.341} \\
\hline & No & $69(95.8 \%)$ & 27 (39.1\%) & $42(60.9 \%)$ & \\
\hline \multirow[t]{2}{*}{ CT confirmed volvulus } & Yes & $44(57.9 \%)$ & $15(34.1 \%)$ & $29(65.9 \%)$ & \multirow[t]{2}{*}{0.392} \\
\hline & No & $32(42.1 \%)$ & $14(43.8 \%)$ & $18(56.2 \%)$ & \\
\hline \multirow[t]{2}{*}{ 360-degree twist } & Yes & $19(24.7 \%)$ & $10(52.6 \%)$ & $9(47.4 \%)$ & \multirow[t]{2}{*}{0.159} \\
\hline & No & $58(75.3 \%)$ & $20(34.5 \%)$ & $38(65.5 \%)$ & \\
\hline \multirow[t]{2}{*}{ Ischaemia on CT } & Yes & $35(48.0 \%)$ & $15(42.9 \%)$ & $20(57.1 \%)$ & \multirow[t]{2}{*}{0.319} \\
\hline & No & $38(52.0 \%)$ & $12(31.6 \%)$ & $26(68.4 \%)$ & \\
\hline \multirow[t]{2}{*}{ Abnormal WCC } & Yes & $33(42.3 \%)$ & $11(33.3 \%)$ & $22(66.7 \%)$ & \multirow[t]{2}{*}{0.322} \\
\hline & No & $45(57.7 \%)$ & $20(44.4 \%)$ & $25(55.6 \%)$ & \\
\hline \multirow[t]{2}{*}{ Abnormal Neutroph. } & Yes & $32(41.0 \%)$ & $10(31.3 \%)$ & $22(68.7 \%)$ & \multirow[t]{2}{*}{0.201} \\
\hline & No & $46(59.0 \%)$ & $21(45.7 \%)$ & $25(54.3 \%)$ & \\
\hline \multirow[t]{2}{*}{ Abnormal CRP } & Yes & $46(59.0 \%)$ & $13(28.3 \%)$ & $33(71.7 \%)$ & \multirow[t]{2}{*}{0.013} \\
\hline & No & $32(41.0 \%)$ & $18(56.3 \%)$ & $14(43.7 \%)$ & \\
\hline \multirow[t]{2}{*}{ Admissions > 3} & Yes & $32(41.0 \%)$ & 19 (61.3\%) & $13(27.7 \%)$ & \multirow[t]{2}{*}{0.003} \\
\hline & No & 46 (59.0\%) & $12(38.7 \%)$ & $34(72.3 \%)$ & \\
\hline
\end{tabular}

Essentially, female patients and those with normal CRP are more prone to undergo surgery. Furthermore, patients are more likely to undergo a surgical operation within their first 3 admissions for volvulus. In fact, the curve of the proportional hazard to undergo surgery is particularly steep in the first phase (Fig. 1).

Multivariate analysis by binary logistic regression confirmed gender, CRP and admissions greater than 3 as independent prognostic factors (Table 2). 
Table 2

Multivariate analysis. Dependent variable: surgery.

\begin{tabular}{|llll|}
\hline Factor & Beta coefficient & Odds ratio & $P$ \\
\hline Gender (male) & -1.20 & 0.30 & 0.036 \\
\hline Admissions $>3$ & 1.47 & 4.37 & 0.005 \\
\hline Abnormal CRP & -1.12 & 0.33 & 0.033 \\
\hline Intercept & 0.38 & & \\
\hline Overall model fit: Chi-square $=18.1$ - Degrees of freedom $=3-\mathrm{p}<0.001$ \\
\hline
\end{tabular}

Median follow-up was 15.2 months (range 1-133). Overall mean survival was $54.9 \pm 8.8$ months since the first admission and was significantly better in patients who did have an operation than in those who did not $(68.6 \pm 7.2$ vs $31.4 \pm 7.8, p<0.001)$, but did not change significantly according to timing of surgery, either within the first 2 admissions or afterwards (Fig. 2). Survival was also better in patients with low Social Score (Fig. 3).

However, the actual difference is mostly in the first 12 months of the survival curve as in the long time the curves tend to converge. Quite expectedly, survival was longer in younger patients (Fig. 4) and was worse in man with respect to women (Fig. 5).

Cox regression analysis confirmed only gender (male) and surgery as independent prognostic factors (Table 3).

Table 3

Multivariate analysis of survival (Cox ProportionalHazards Regression).

\begin{tabular}{|lll|}
\hline Factor & Beta coefficient & $\boldsymbol{P}$ \\
\hline Gender (male) & 1.228 & 0.026 \\
\hline Surgery & -2.012 & 0.0002 \\
\hline Overall model fit: & Chi-square $=30.756-p<0.0001$ \\
\hline
\end{tabular}

\section{Discussion}

Sigmoid volvulus is a frequent reason for emergency surgical admission. Despite Literature evidence and guidelines, the usual emergency treatment is bowel decompression and derotation, but at the price of a high rate of recurrence that in our series is about $75 \%$, which is consistent with the available Literature $[4-7,11,12]$. Mortality has been shown to be up to $20 \%$ during recurrence and so endoscopic devolution must be considered only a temporary measure $[7,13]$ leading to a definitive treatment where possible. 
Unfortunately, guidance on indications and timing of surgery are not very clear, and the decision remains with the emergency surgeon and the patient whose ability to understand, retain and use the information given may be impaired due to their chronic and acute illness.

Patients with normal CRP are more likely to undergo surgery. This finding can be related to a strict selection of patients, trying to avoid surgery in those patients with sepsis and therefore with high risk of mortality, as demonstrated by Heo et al. [14]. In fact, while for other conditions sepsis would prompt an emergency operation for source control, in already frail and looked-after patients, sepsis can be a terminal event and surgery can be considered futile.

Patients are more likely to undergo surgery within their first three admissions. This may relate to the patient's fitness for surgery and/or to their complicated presentation. The ASCRS guidelines advised that after a single episode of volvulus elective surgery should be planned to prevent further recurrence [8], but not all patients are considered fit for surgery at their first admission. Despite the presence of objective criteria, fitness for surgery is often a matter of subjective evaluation that can be biased by personal ideas and impressions. As a matter of fact, in our series the choice of surgery was not influenced by the ASA score (Table 1), thus demonstrating that at the moment the surgical choice is still based on not-betterspecified "clinical criteria". On the contrary, we feel that surgery must be considered the first choice in patients whose fitness has been evaluated by strict evidence-based criteria, also considering the overall low surgical risk and the low risk of recurrence after surgical resection [7].

Quénéhervé et al. found that patients in their 'no surgery group' were older and frailer and agree that surgeons are more reluctant to carry our colonic surgery on this cohort of patients, therefore, quite expectedly, general conditions of the patients - and their frailty - may represent a factor to be considered when deciding the treatment strategy [3].

The timing of planned surgery remains controversial. Some suggest that definitive surgery should be carried out within 2-5 days of the initial volvulus $[15,16]$. Furthermore Johansson et al. found that recurrence was more frequent after the second episode, leading us to believe that elective surgery should only be advised following the second recurrence and not the first [5]. Our series demonstrates that there is no significant difference of long-term survival according to timing of surgery (Fig. 2), therefore early surgery may be suggested against a late operation mostly to improve quality of life and reduce the risk of further admissions.

Long term survival in this cohort of patients strictly depends on the treatment they receive. Overall mean survival was about 5 years, but long-term survival can only be possible in patients who undergo a surgical resection, while only less than $20 \%$ of non-surgical patients are still alive 5 years after their presentation. Ifversen et al. found that patients who were treated surgically after the first occurrence had a far better survival. [4]. Interestingly, in our cohort, survival was not affected by timing of surgery and patients operated after the first two admissions had similar survival than those operated earlier. However, the actuarial curve is not completely overlapping (Fig. 2), allowing us to hypothesize that with a larger 
sample and a longer follow-up it could be possible to highlight an advantage for the patients who had an earlier operation.

Our study shows survival was better in patients with low social score. It is worth specifying that our "social score" is only indirectly related to medical conditions and general frailty, being on the contrary a classification of the social circumstances of the patient. While it is obvious that generally speaking more frail patients may likely need a more complex social support, we wonder if this is enough to justify a shorter survival in patients with high social score, independently of clinical frailty. In fact, in our analysis, frailty has not been found to be an independently prognostic variable. It looks like some frail patients who live independently may have better outcomes with respect to those with the same frailty who need strong social support. This is an interesting issue that should be analysed with a different study design on a larger population.

In our study survival was also better in women. This may be, at least partially, related to the fact that women were more likely to be offered a surgical operation, therefore they might have been in better general conditions. However, this recalls once again the issue of 'clinical perception'. It is possible that women were perceived to be in better general conditions, without using objective criteria but only the surgical "first impression". Another factor must be anyway taken into account, the natural longer survival and the greater resilience of female patients with respect to men [17].

This paper offers a significant insight on a selected cohort and allows to draw interesting conclusions that somewhat challenge the current conservative attitude towards patients treated for sigmoid volvulus, supporting clinical evidence and guidelines. However, beyond its intrinsic value, it can be regarded as a pilot study that can prompt further research. In fact, main limitations of our paper are the small sample size, and the retrospective nature, along with the relatively short follow-up. Although both our cohorts of surgical and non-surgical patients matched for every basic clinical aspect, the surgical decision was not always based on strict clinical criteria but mostly on the choice of the surgeon in charge. A proper randomised controlled trial would be able to clarify some still unsolved issues, such as indications, contraindications and timing of surgery.

\section{Conclusions}

In conclusion, a more aggressive approach to patients with sigmoid volvulus seems to be justified on the ground of the demonstration of a better and longer survival in those who undergo a surgical operation. We suggest that every patient with recurrent sigmoid volvulus is thoroughly assessed for fitness and offered surgery as soon as possible.

\section{Abbreviations}

ASA - American Society of Anaesthesiologists

ASCRS - American Society of Colon and Rectal Surgeons 
CRP - C-Reactive Protein

CT - computer tomography

STROBE - Strengthening the Reporting of Observational Studies in Epidemiology.

\section{Declarations}

Ethics approval and consent to participate - This is a retrospective study on anonymised data so ethical approval is considered unnecessary.

Consent for publication - No individual person's data is included in this study.

Availability of data and materials - The dataset used and/or analysed during the current study are available from the corresponding author on reasonable request.

Competing interests - The authors declare that they have no competing interests.

Funding - No funding was available for this study.

Author's contributions - ZS, GBS and GDT conceived the study. MS. RS, RA, RA, DA, AD, MP and AS retrieved data. ZS and GDT analysed the data. ZS, MS and GDT drafted the manuscript. ZS, MS, RA, RA, $D A, A D, M P, A S, G B S$ and GDT discussed the analysis and approved the final version of the manuscript and are equally accountable for the content of this study.

Acknowledgments - not applicable

\section{References}

1. Ballantyne GH. Review of sigmoid volvulus: history and results of treatment. Dis Colon Rectum. 1982;25(5):494-501.

2. Halabi WJ, Jafari MD, Kang CY, Nguyen VQ, Carmichael JC, Mills S, et al. Colonic volvulus in the United States: trends, outcomes, and predictors of mortality. Ann Surg. 2014;259:293-301.

3. Quénéhervéa L, Dagouat C, Le Rhunb M, Robles E, P-C, Duchalais E, des Varannes SB, Touchefeua Y, Chapellea N, Coron E. Outcomes of first-line endoscopic management for patients with sigmoid volvulus. Dig Liver Dis. 2018;51:386-90.

4. Ifversen AK, Kjaer DW. More patients should undergo surgery after sigmoid volvulus. World J Gastroenterol. 2014;20:18384-9.

5. Johansson N, Rosemar A, Angenete E. Risk of recurrence of sigmoid volvulus: a single-centre cohort study. Colorectal Dis. 2018;20:529-35.

6. Larkin JO, Thekiso TB, Waldron R, Barry K, Eustace PW. Recurrent sigmoid volvulus - early resection may obviate later emergency surgery and reduce morbidity and mortality. Ann R Coll Surg Engl. 
2009;91:205-9.

7. Bruzzi E, Lefèvre JH, Desaint B, Nion-Lamruier I, Bennis M, Chafai N, Tiret E, Parc Y. Management of acute sigmoid volvulus: short- and long-term results. Colorectal Dis. 2015;17:922-8.

8. Vogel JD, Feingold DL, Stewart DB, et al. Clinical practice guidelines for colon volvulus and acute colonic pseudo-obstruction. Dis Colon Rectum. 2016;59:589-600.

9. Rockwood K, Song X, MacKnight C, Bergman H, Hogan DB, McDowell I, Mitnitski A. A global clinical measure of fitness and frailty in elderly people. CMAJ. 2005 Aug 30;173:489 - 95.

10. STROBE Guidelines. https://www.strobe-statement.org/index.php?id=strobe-home. Last accessed 14th February 2021.

11. Hougaard HT, Qvist N. Elective surgery after successful endoscopic decompression of sigmoid volvulus may be considered. Dan Med J. 2013;60:A4660.

12. Yassaie 0 , Thompson-Fawcett M, Rossaak J. Management of sigmoid volvulus: is early surgery justifiable? ANZ J Surg. 2013;83:74-8.

13. Naveed M, Jamil LH, Fuji-Lau LL, et al. American Society for Gastrointestinal Endoscopy guideline on the role of endoscopy in the management of acute colonic pseudo-obstruction and colonic volvulus. Gastrointest Endosc. 2020;91:228-35.

14. Heo S, Kim HJ, Oh BJ, et al. Sigmoid volvulus: identifying patients requiring emergency surgery with the dark torsion knot sign. Eur Radiol. 2019;29:5723-30.

15. Tsai MS, Lin MT, Chang KJ, Wang SM, Lee PH. Optimal interval from decompression to semi-elective operation in sigmoid volvulus. Hepatogastroenterology. 2006;53:354-6.

16. Perrot L, Fohlen A, Alves A, et al. Management of the colonic volvulus in 2016. J Visc Surg. 2016;153:183-92.

17. Khircchoff P, Dincler S, Buchmann P. A multivariate analysis of potential risk factors for intra- and postoperative complications in 1316 elective laparoscopic colorectal procedures. Ann Surg. 2008;248:259-65.

\section{Figures}




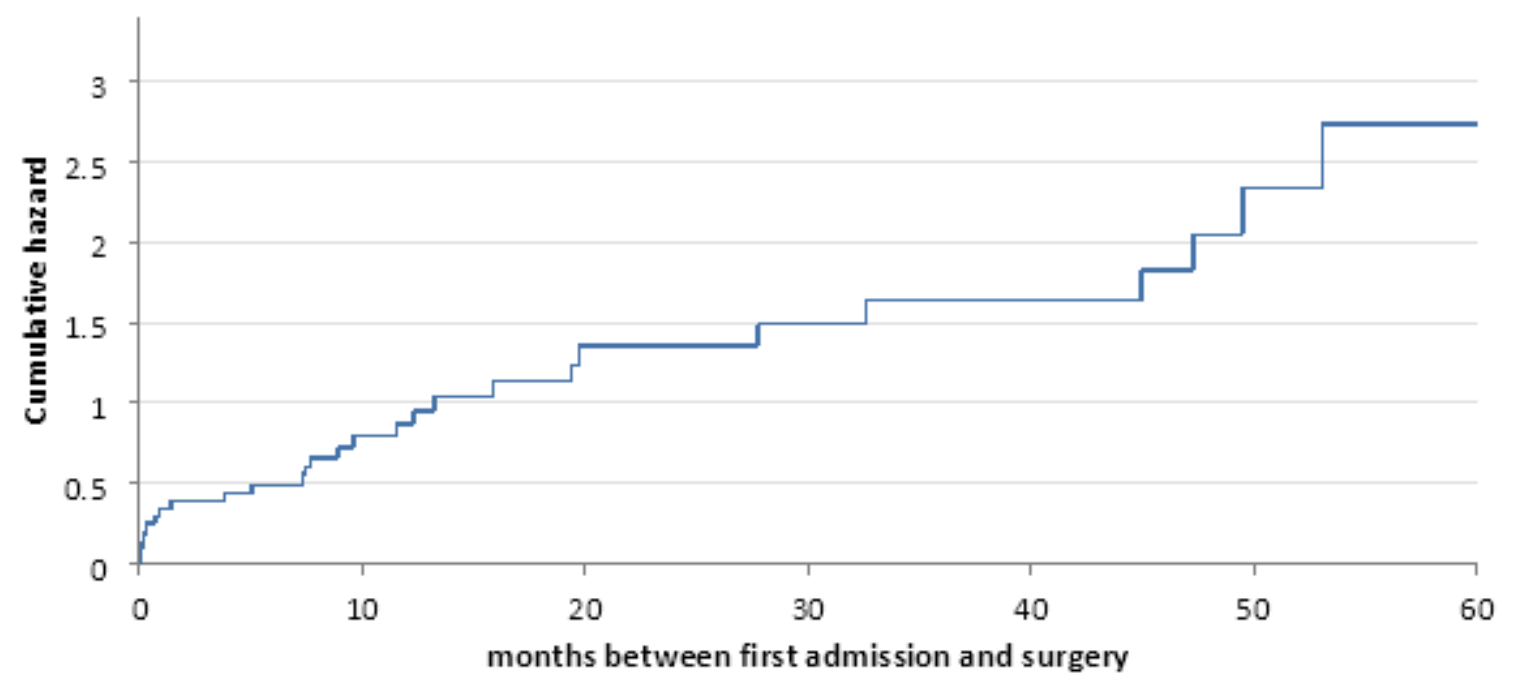

Figure 1

Proportional hazard to undergo surgery.

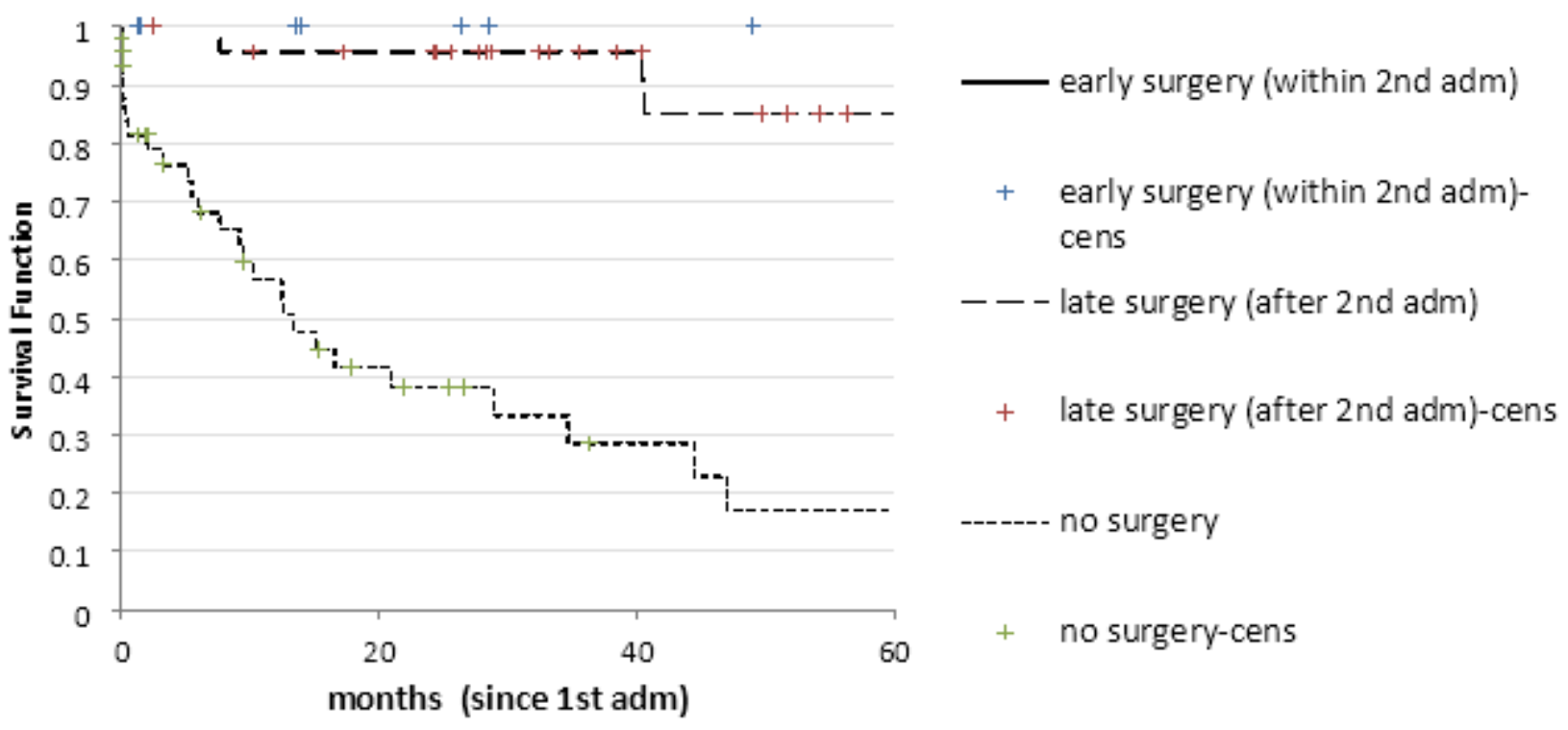

Figure 2

Survival according to treatment (early surgery vs late surgery vs no surgery) $(p<0.001)$. 


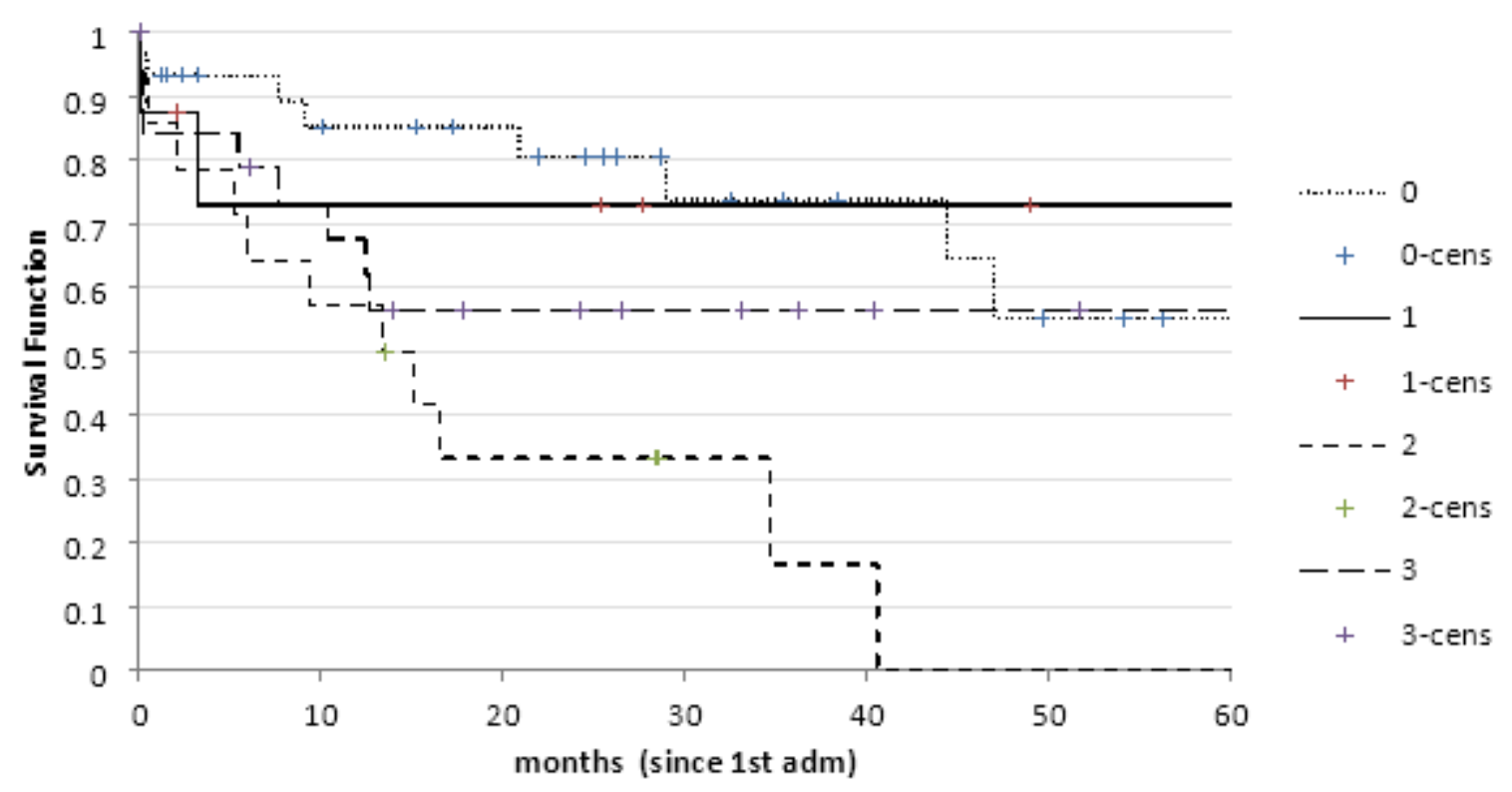

Figure 3

Survival according to Social Score $(p<0.01)$ (for definition of Social Score see text).

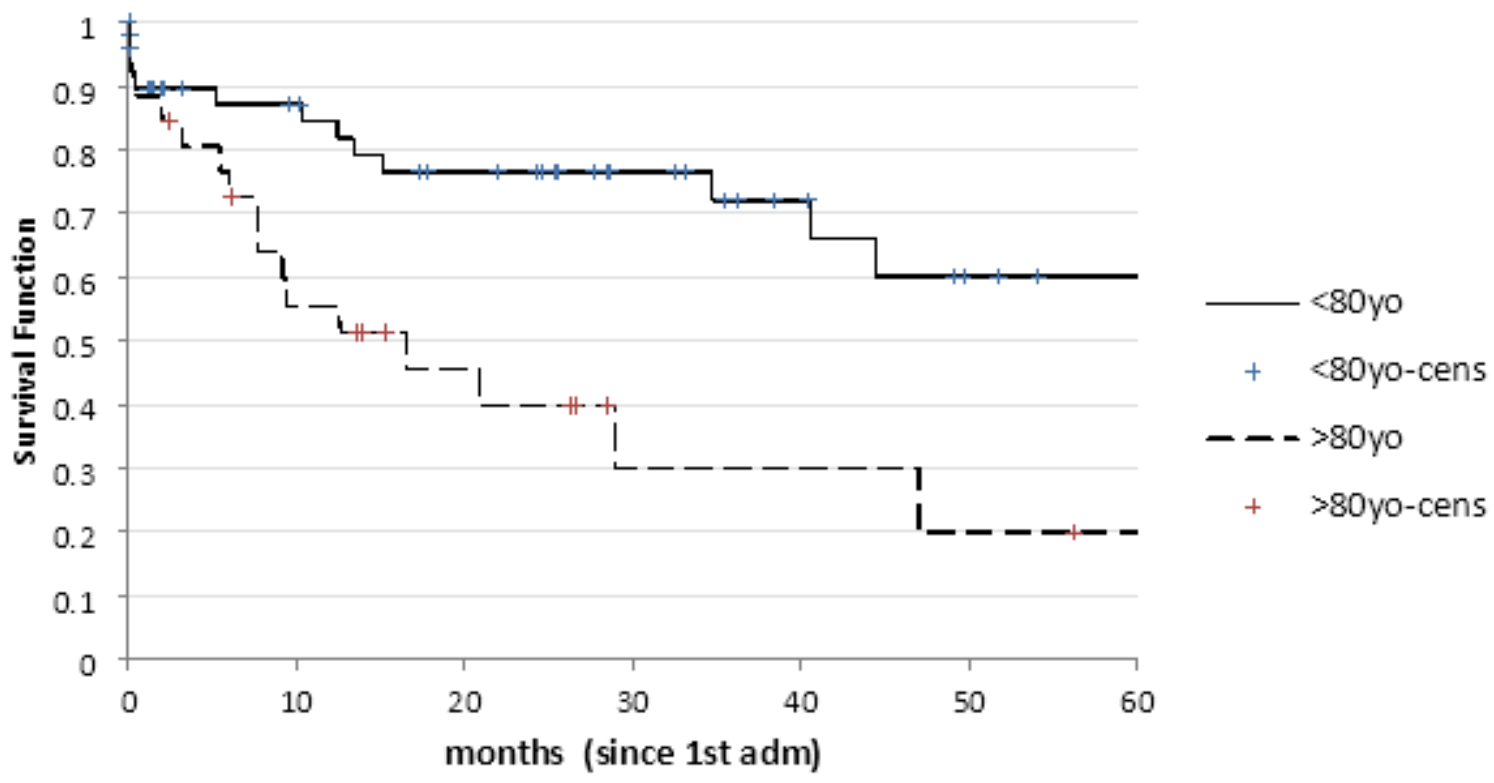

Figure 4

Survival according to age $(>$ or $<80)(p<0.003)$. 


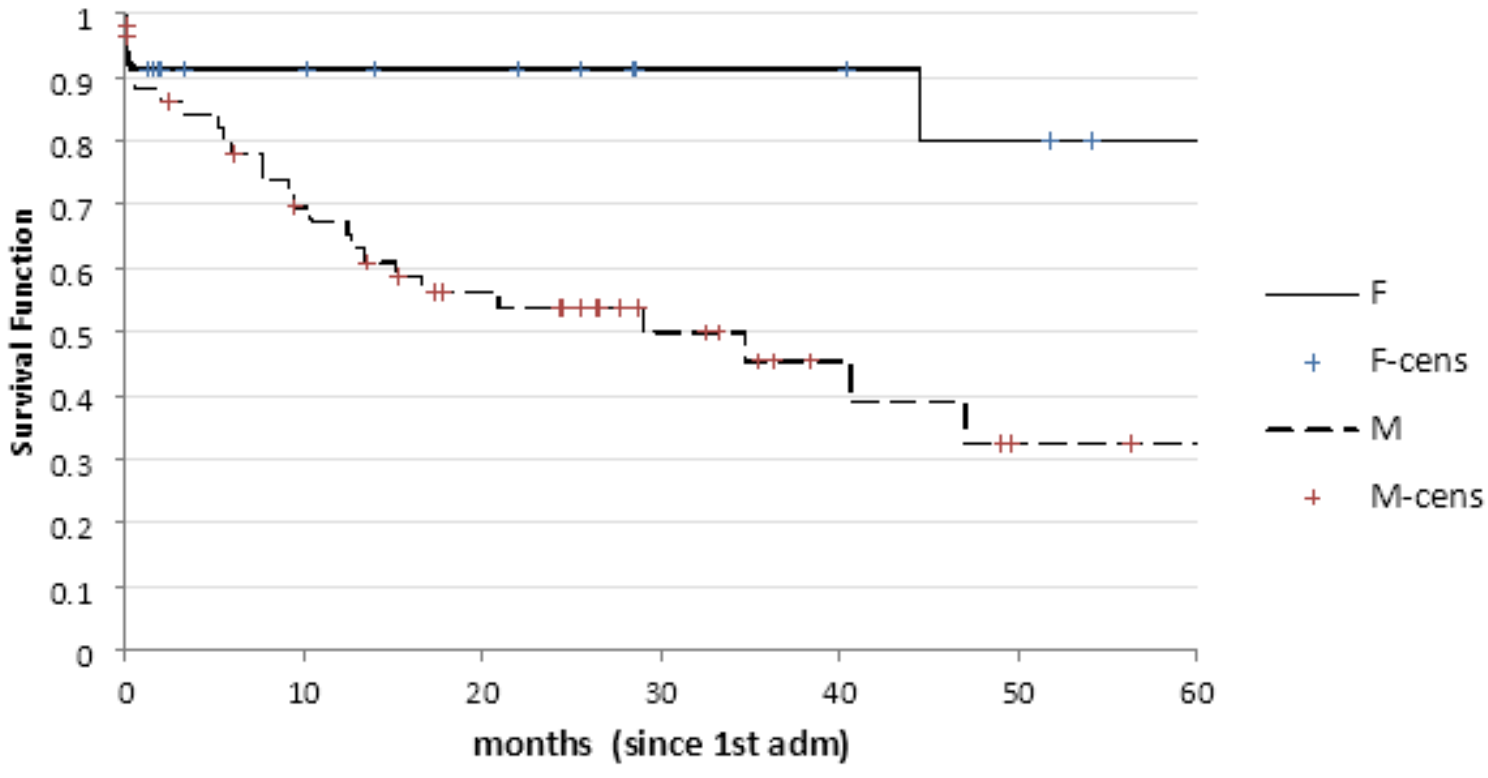

Figure 5

Survival according to gender $(\mathrm{p}<0.005)$. 